\title{
Smoothened-activating lipids drive resistance to CDK4/6 inhibition in Hedgehog-associated medulloblastoma cells and preclinical models
}

\author{
Vikas Daggubati, ${ }^{1,2,3,4}$ Jordan Hochstelter, ${ }^{1,2}$ Anirudh Bommireddy, ${ }^{1,2}$ Abrar Choudhury, ${ }^{1,2,3,4}$ Alexis Leigh Krup, ${ }^{1}$ Pervinder Kaur, \\ Pakteema Tong, ${ }^{5}$ Amy Li, ${ }^{5}$ Libin Xu, ${ }^{5}$ Jeremy F. Reiter, ${ }^{6}$ and David R. Raleigh ${ }^{1,2,3}$ \\ 'Department of Radiation Oncology, ${ }^{2}$ Department of Neurological Surgery, ${ }^{3}$ Biomedical Sciences Graduate Program, and ${ }^{4}$ Medical Scientist Training Program, UCSF, San Francisco, California, USA. \\ ${ }^{5}$ Department of Medicinal Chemistry, University of Washington, Seattle, Washington, USA. ${ }^{6}$ Department of Biochemistry and Biophysics, Cardiovascular Research Institute, Chan Zuckerberg Biohub, UCSF, \\ San Francisco, California, USA.
}

\begin{abstract}
Medulloblastoma is an aggressive pediatric brain tumor that can be driven by misactivation of the Hedgehog $(\mathrm{HH})$ pathway. CDK6 is a critical effector of oncogenic $\mathrm{HH}$ signaling, but attempts to target the HH pathway in medulloblastoma have been encumbered by resistance to single-agent molecular therapy. We identified mechanisms of resistance to CDK6 inhibition in $\mathrm{HH}$-associated medulloblastoma by performing orthogonal CRISPR and CRISPR interference screens in medulloblastoma cells treated with a CDK4/6 inhibitor and RNA-Seq of a mouse model of $\mathrm{HH}$-associated medulloblastoma with genetic deletion of Cdk6. Our concordant in vitro and in vivo data revealed that decreased ribosomal protein expression underlies resistance to CDK6 inhibition in HH-associated medulloblastoma, leading to ER stress and activation of the unfolded protein response (UPR). These pathways increased the activity of enzymes producing Smoothened-activating (SMO-activating) sterol lipids that sustained oncogenic $\mathrm{HH}$ signaling in medulloblastoma despite cell-cycle attenuation. We consistently demonstrated that concurrent genetic deletion or pharmacological inhibition of CDK6 and HSD11ß2, an enzyme producing SMO-activating lipids, additively blocked cancer growth in multiple mouse genetic models of $\mathrm{HH}$-associated medulloblastoma. Our data reveal what we believe to be a novel pathway of resistance to CDK4/6 inhibition as well as a novel combination therapy to treat the most common malignant brain tumor in children.
\end{abstract}

\section{Introduction}

Hedgehog $(\mathrm{HH})$ signaling is essential for development and adult tissue homeostasis (1). Misactivation of the $\mathrm{HH}$ pathway is associated with congenital disorders and cancers, such as medulloblastoma and basal cell carcinoma, the most common cancer in the United States (2). Vertebrate HH signals are transduced through the primary cilium, an antenna that projects from the surface of most cells (3). Upon pathway activation, Smoothened (SMO) accumulates in the primary cilium and activates the GLI family of transcription factors, which travel to the nucleus and activate the $\mathrm{HH}$ gene expression program. SMO inhibition has improved the treatment of adult basal cell carcinoma (4) but has not improved the treatment of pediatric medulloblastoma because of acquired resistance $(5,6)$ and toxicity (7). Current therapies for medulloblastoma, such as cytotoxic chemotherapy and ionizing radiation, cause secondary cancers and permanent endocrine, neurologic, and cognitive dysfunctions $(8,9)$. Thus, there is an urgent, unmet need for new therapies to treat pediatric patients with medulloblastoma.

Conflict of interest: The authors have declared that no conflict of interest exists. Copyright: () 2021, American Society for Clinical Investigation.

Submitted: June 8, 2020; Accepted: January 14, 2021; Published: March 15, 2021.

Reference information: J Clin Invest. 2021;131(6):e141171.

https://doi.org/10.1172/JCl141171
We have shown that CDK6, an enzyme critical for $\mathrm{G}_{1}$-phase progression through the cell cycle (10), is a direct transcriptional target of oncogenic $\mathrm{HH}$ signaling and that genetic or pharmacologic inhibition of CDK6 blocks the growth of $\mathrm{HH}$-associated medulloblastomas (11). Small-molecule CDK4/6 inhibitors have revolutionized metastatic breast cancer treatment (10), and clinical trials exploring the efficacy of CDK4/6 inhibitors in medulloblastoma are underway (NCT04238819, NCT03434262, and NCT01878617). Nevertheless, acquired resistance to molecular monotherapy is common in medulloblastoma $(5,6)$ and breast cancer (10), suggesting that CDK4/6 inhibitors may be most effective in combination with other therapies that block resistance mechanisms, which remain unknown in medulloblastoma.

\section{Results and Discussion}

To identify the mechanisms underlying resistance to CDK4/6 inhibitors in $\mathrm{HH}$-associated medulloblastoma, cellular and biochemical mechanisms of $\mathrm{HH}$ signal transduction were studied in DAOY, UW228, and ONS76 human medulloblastoma cells (12). Compared with DAOY cells, the majority of UW228 cells lacked primary cilia (Supplemental Figure 1A; supplemental material available online with this article; https://doi.org/10.1172/ JCI141171DS1), and ONS76 cells expressed shortened cilia (Supplemental Figure 1B), a marker of reduced HH signal transduction (13-15). When stimulated with recombinant Sonic Hedgehog 
(SHH), neither UW228 nor ONS76 cells accumulated SMO in cilia (Supplemental Figure 1, C and D) or activated the HH transcriptional program (Supplemental Figure 1, E and F) to the same extent as DAOY cells. Further, abemaciclib, a clinical CDK4/6 inhibitor that penetrates the blood-brain barrier (10), blocked clonogenic growth of DAOY cells in a dose-dependent manner (Supplemental Figure $1, \mathrm{G}$ and $\mathrm{H})$. Given these findings, we generated DAOY cells stably expressing Cas9 or dCas9-KRAB for genome-wide resistance screens (Supplemental Figure 1I). The complementary Cas9 and dCas9-KRAB systems rely on sgRNAs to generate dsDNA breaks and inactivate genes, or to sterically and epigenetically inhibit gene transcription, respectively (Figure 1A). To validate DAOYCas9 and DAOY ${ }^{\mathrm{dCas} 9-\mathrm{KRAB}}$ cells, we targeted CDK6 using sgRNAs that attenuated clonogenic growth compared with cells transduced with nontargeting sgRNAs (sgNTCs) (Supplemental Figure 1, J-L). Thus, DAOY $^{\text {Cas9 }}$ and DAOYdCas9-KRAB cells were transduced with genomewide sgRNA libraries and cultured cells in abemaciclib for 10 days to enrich for resistant clones (Figure 1B). Genes conferring resistance to $\mathrm{CDK} 4 / 6$ inhibition in $\mathrm{HH}$-associated medulloblastoma cells were identified by sgRNA sequencing and comparison of sgRNA enrichment between DAOYCas9 or DAOY ${ }^{\mathrm{dCas9-KRAB}}$ cells treated with abemaciclib or vehicle control (Supplemental Table 1).

In breast cancer, resistance to CDK4/6 inhibition is mediated by the induction of $\operatorname{CDK} 6(10,16,17)$, but gene ontology (GO) analyses in DAOY ${ }^{\mathrm{dCas} 9-\mathrm{KRAB}}$ and 2 independent DAOY ${ }^{\mathrm{Cas} 9}$ abemaciclib resistance screens identified enrichment of sgRNAs targeting ribosome and ribosome biogenesis genes (Figure 1C, Supplemental Figure 2, A and B, and Supplemental Table 1). CDK6 expression did not increase in genome-wide enrichment screens (Supplemental Figure 2C), or with conditioned resistance to abemaciclib in parental DAOY cells (Supplemental Figure 2D), suggesting that the resistance mechanisms previously reported in breast cancer were not present in our medulloblastoma samples. To validate these results in vitro, we transduced DAOY ${ }^{\mathrm{dCas} 9-\mathrm{KRAB}}$ cells with sgRNAs targeting the top 24 genes from our CRISPR interference (CRISPRi) abemaciclib resistance screen and performed clonogenic assays to confirm that stable suppression of these genes promoted resistance to $\mathrm{CDK} 4 / 6$ inhibition in $\mathrm{HH}$-associated medulloblastoma cells (Supplemental Figure 3). To validate these results in vivo, we used a conditional mouse genetic model that relies on Cre recombinase under the control of Math1 regulatory sequences to express a constitutively active, oncogenic point mutation of Smo $\left(S m o M 2^{c}\right)$ in cerebellar external granule layer cells that give rise to $\mathrm{HH}$-associated medulloblastoma $(18,19)$. Medulloblastomas were isolated from six Math1-Cre SmoM2 ${ }^{C} \mathrm{Cdk} 6^{\mathrm{KO} / \mathrm{KO}}$ mice for RNA-Seq and differential expression analysis compared with 3 Math1-Cre SmoM2 ${ }^{c} C_{d k 6^{W T / W T}}$ medulloblastomas (ref. 11 and Supplemental Table 2). The majority of differentially expressed genes were suppressed with loss of Cdk6 (Supplemental Figure 4A), and, consistent with genome-wide resistance screens in vitro, GO analyses revealed that ribosome and ribosome biogenesis genes dominated suppressed transcripts in Math1-Cre SmoM2 ${ }^{C} C d k 6^{K O / K O}$ medulloblastomas compared with Math1-Cre SmoM2 ${ }^{C} C d k 6^{W T / W T}$ tumors (Supplemental Figure 4B and Supplemental Table 2).

Inactivating mutations in ribosome genes can drive cancer (20), and genome-wide screens demonstrate functional clustering of ribosome subunits (21), suggesting that loss of ribosome genes may have similar phenotypic consequences. Our in vitro and in vivo data converged on the suppression of RPL1O and $R P L 23 A$ as drivers of resistance to CDK4/6 inhibition (Figure 1D and Supplemental Table 1), and RPL1O substitutions are found in approximately $10 \%$ of $\mathrm{T}$ cell acute lymphoblastic leukemias and multiple myelomas $(22,23)$. Thus, to validate the function of RPL10 in HH-associated medulloblastoma, we suppressed RPL1O

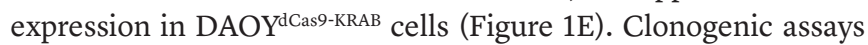
of stable cell lines treated with abemaciclib revealed that loss of RPL1O enhanced clonogenic growth compared with DAOY ${ }^{\text {dCas9-KRAB }}$ cells expressing sgNTCs (Figure 1F). Moreover, expression of RPL1O and other ribosome biogenesis genes was suppressed in Math1-Cre SmoM2 ${ }^{C}$ Cdk6 ${ }^{\mathrm{KO} / K O}$ medulloblastomas compared with expression in Math1-Cre SmoM2 ${ }^{C}$ Cdk6 $6^{W T / W T}$ tumors (Figure 1G, Supplemental Figure 4C, and Supplemental Table 2).

Cellular functions of RPL10 are poorly understood (24), but ribosomes and ribosome biogenesis are critical for lipid homeostasis (25). Indeed, inhibition of ribosome biogenesis causes ER stress (26), which activates the unfolded protein response (UPR) and stimulates lipogenesis $(27,28)$. Our laboratory and others have shown that sterol and oxysterol lipids bind to SMO and activate the $\mathrm{HH}$ transcriptional program $(29,30)$. Thus, ribosome gene suppression may activate the UPR to increase cellular levels of SMO-activating lipids and drive resistance to CDK4/6 inhibition in $\mathrm{HH}$-associated medulloblastomas. To test this hypothesis, ribosome biogenesis, UPR activation, and SMO-activating lipid levels were quantified in DAOY ${ }^{\mathrm{dCas} 9-\mathrm{KRAB}}$ cells stably expressing sgRNAs targeting RPL1O or sgNTCs (Figure 1E). Quantitative reverse transcriptase PCR (qRT-PCR) revealed that suppression of RPL1O inhibited ribosome biogenesis, as measured by decreased expression of ribosome precursor rRNAs, and activated the UPR, as measured by splicing of the master transcription factor XBP1 (Figure $1 \mathrm{H}$ and ref. 27). Immunoblots demonstrated that suppression of RPL1O also increased phosphorylation of eIF $2 \alpha$ and expression of ATF4 (Figure 1I), both of which are hallmarks of UPR activation (31). Immunofluorescence confocal microscopy using perfringolysin O (PFO), a bacterial toxin that labels the SMO agonist cholesterol in live cells (29), further showed that suppression of RPL1O increased SMO-activating lipid levels in $\mathrm{HH}$-associated medulloblastoma cells (Figure 1, J and $\mathrm{K}$ ).

The UPR activates parallel signaling pathways to coordinate diverse cellular responses, including IRE-1 activation and $X B P 1$ splicing; and PERK activation of eIF $\alpha$, phosphorylated eIF $\alpha$ (p-eIF $\alpha$ ), and ATF (32). Suppression of ribosome and ribosome biogenesis activated IRE-1/XBP1 and PERK signaling in HH-associated medulloblastoma cells (Figure 1, H and I), and IRE-1/XBP1 signaling is canonically associated with UPR-mediated lipogenesis (32). Thus, to define the biochemical mechanisms that increase SMO-activating lipids in $\mathrm{HH}$-associated medulloblastoma cells after loss of ribosome genes, we treated DAOY ${ }^{\mathrm{dCas} 9-\mathrm{KRAB}}$ cells stably expressing sgNTCs or sgRNAs suppressing RPL1O with the IRE-1 inhibitor KIRA6, the PERK inhibitor GSK2606414, or vehicle control. Immunofluorescence confocal microscopy using PFO showed that pharmacologic inhibition of IRE-1/XBP1 signaling blocked SMO-activating lipid induction in response to RPL1O suppression (Figure 1L) but that pharmacologic inhibition of PERK did not (Supplemental Figure 5A). 
A

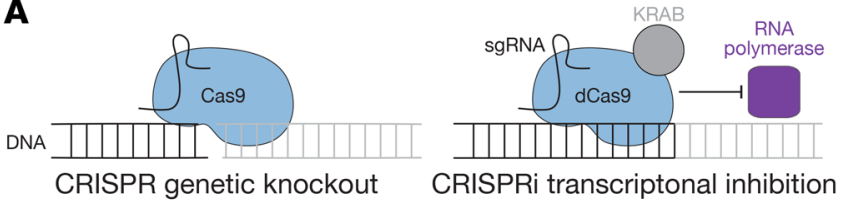

B

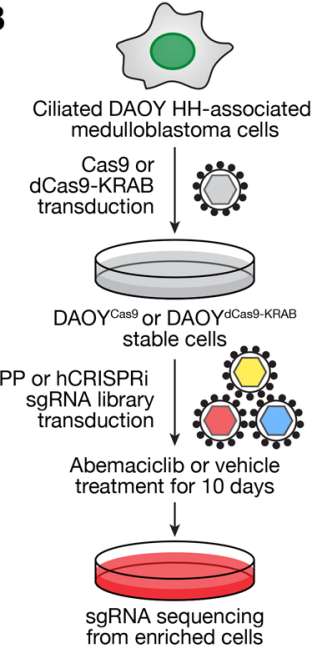

C

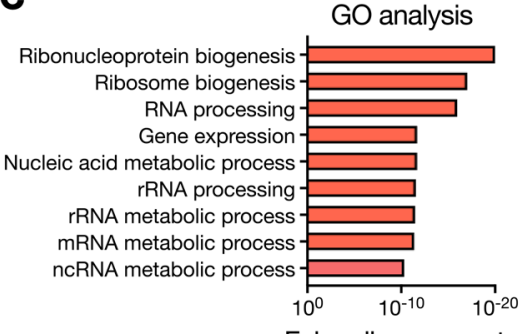

False discovery rate

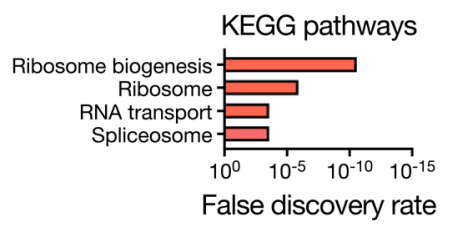

False discovery rate

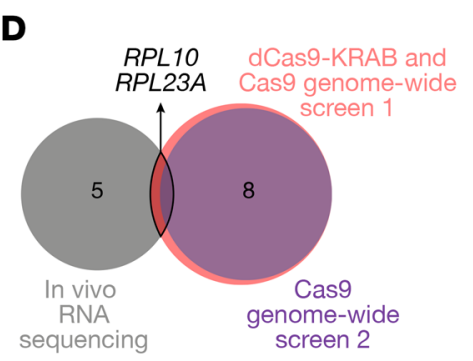

$\mathbf{F}$

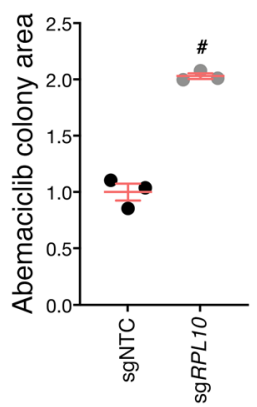

DAOYdCas9-KRAB

G
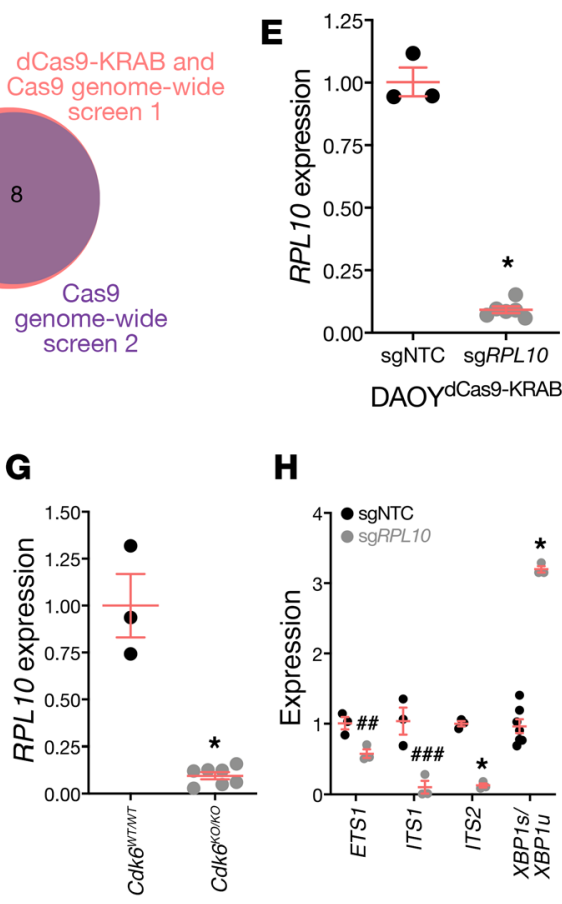

Math1-Cre SmoM2
H

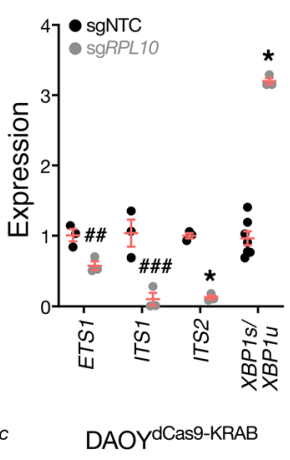

I

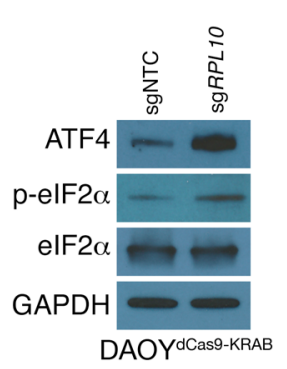

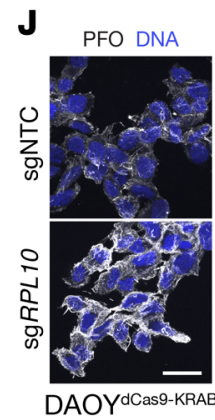

$\mathbf{K}$

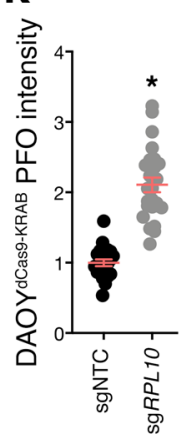

$\mathbf{L}$

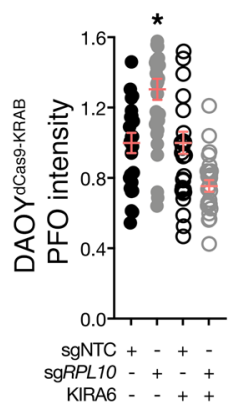

M

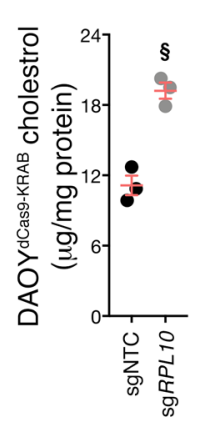

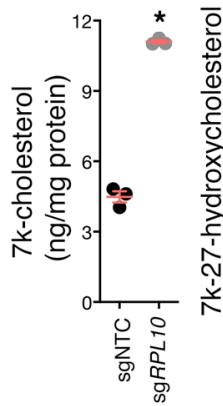

Figure 1. Suppression of ribosome biogenesis drives resistance to CDK4/6 inhibition in HH-associated medulloblastoma by inducing SMO-activating lipid synthesis. (A) Schematic of CRISPR and CRISPRi gene suppression through dsDNA breaks and steric inhibition, respectively. (B) Genome-wide CDK4/6 inhibition resistance screen study design for $\mathrm{HH}$-associated medulloblastoma cells. GPP, Genetic Perturbation Platform; hCRISPRi, human CRISPRi. (C) Suppressed pathways in DAOYCas9 $(n=2)$ and DAOYdas9-KRAB $(n=1)$ genome-wide CDK4/6 inhibition resistance screens. (D) Venn diagram of suppressed ribosome and ribosome biogenesis genes mediating the resistance to loss of CDK6 in $\mathrm{HH}$-associated medulloblastoma in vitro and in vivo. Numbers indicate overlapping genes. Among these, only RPL10 and RPL23A were implicated in both in vitro and in vivo experiments. Non-ribosome genes were omitted from this analysis. (E) qRT-PCR assessment of $R P L 10$ suppression in DAOY ${ }^{\text {dCas9-KRAB }}$ cells. ${ }^{*} P<0.0001 . n=2$. (F) DAOYdCas9-KRAB clonogenic assays after 10 days of abemaciclib treatment demonstrated that $R P L 10$ suppression conferred resistance to CDK4/6 inhibition. ${ }^{*} P=0.0009$. $n=2$. (C) RNA-Seq differential expression analysis of mouse medulloblastomas. ${ }^{*} P<0.0001 . n=9$. (H) qRT-PCR assessment of ribosome precursor rRNAs and the ratio of spliced $X B P 1$ to unspliced XBP1 (XPB1s/XPB1u) in DAOY dCas9-KRAB cells. ${ }^{\#} P=0.008,{ }^{\# \#} P=0.006$, and ${ }^{*} P<0.0001 . n=3$. (I) Immunoblot assessment of UPR activation through phosphorylation of elF2 $\alpha$ and induction of ATF4 in DAOYdCas9-KRAB cells. (J) Microscopic images of PFO cholesterol (white) and DNA (blue) staining in DAOY ${ }^{\text {dCas9-KRAB }}$ cells. Scale bar: $100 \mu \mathrm{m}$. (K) PFO cholesterol staining and quantification in DAOYdCas9-KRAB cells. ${ }^{*} P<0.0001$. $n=2$, with more than 40 cells counted per experiment. (L) PFO cholesterol staining and quantification in DAOYdCas9-KRAB cells after treatment with KIRA6, a UPR inhibitor that blocks IRE-1 upstream of lipogenic signaling. ${ }^{*} P<0.0001 . n=2$, with more than 40 cells counted per experiment. (M) Lipidomic mass spectrometry for $S M 0$-activating lipids and precursor lipids $(7 \mathrm{k}-\mathrm{C})$ in DAOY ${ }^{d C a s 9-K R A B}$ cells. ${ }^{\$} P=0.0017,{ }^{*} P<0.0001,{ }^{\S} P=0.0091$, and ${ }^{* *} P=0.0015$. Data are shown as the mean $\pm \mathrm{SEM}$. A Student's $t$ test was used for statistical comparisons. $n=3$.

To determine whether loss of other ribosome genes induced the UPR or SMO-activating lipids, we transduced DAOYdaas-KRAB cells with sgRNAs suppressing RPL23A (Supplemental Figure 5B), which activated the UPR (Supplemental Figure 5C) and increased SMO-activating lipid levels (Supplemental Figure 5D). Ribosomes fulfill essential but semiredundant cellular functions (21), and in contrast to RPL10 suppression, RPL23A suppression decreased DAOY viability (Supplemental Figure $5 \mathrm{E}$ ), which prevented the functional assessment of resistance to CDK4/6 inhibitors. RPL1O and RPL23A suppression also decreased UW228 and ONS76 viability (Supplemental Figure 5F) and activated the UPR in ONS76 cells but not UW228 cells (Supplemental Figure 5, G-L). Although 
A
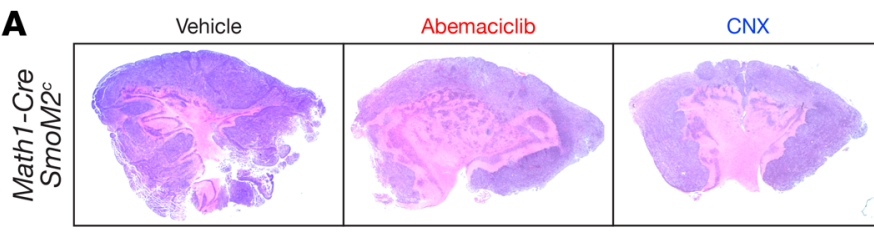

Abemaciclib + CNX

B

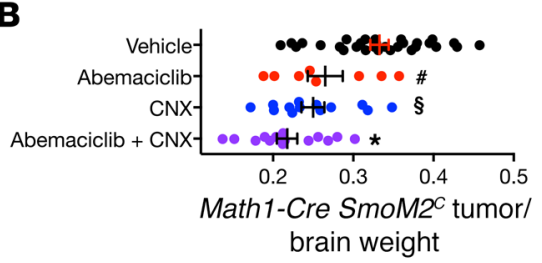

C

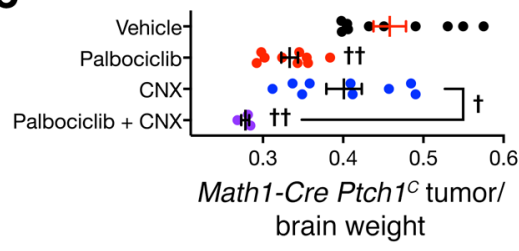

D

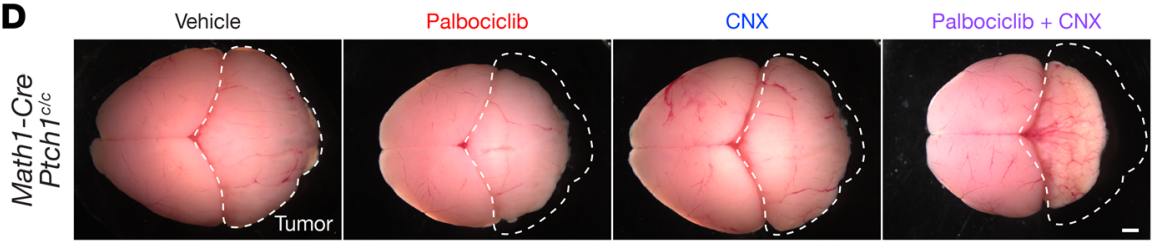

E

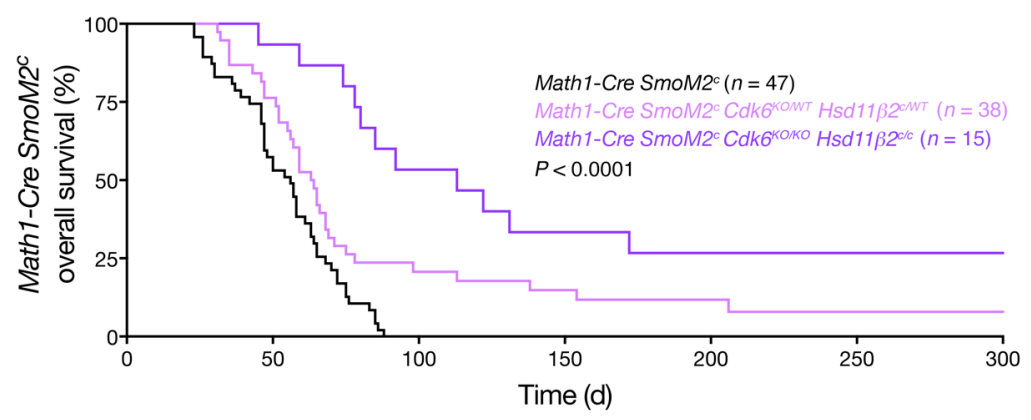

Figure 2. Combination molecular therapy blocking CDK4/6 and SMO-activating lipid synthesis attenuates the growth of HH-associated medulloblastomas. (A) H\&E staining of sagittal mouse medulloblastoma sections after 14 days of treatment, from P21 to P35. Scale bar: $1 \mathrm{~mm}$. (B) Quantification of P35 tumor to brain weight ratios for Math1-Cre SmoM2 ${ }^{\complement}$ mice. Data are shown as the mean $\pm \mathrm{SEM}$. ${ }^{\#} P=0.02,{ }^{\circledR} P=$ 0.0003 , and ${ }^{*} P<0.0001 . n=59$. (C) Quantification of P35 tumor to brain weight ratios for Math1-Cre Ptch1/c/C mice. Data are shown as the mean \pm SEM. ${ }^{\dagger} P=0.01$ and ${ }^{\dagger+} P=0.0002 . n=32$. (D) P35 mouse medulloblastoma brain micrographs. Dashed lines represent the vehicle control tumor area overlaid onto other treatment conditions. Scale bar: $5 \mathrm{~mm}$. (E) Kaplan-Meier survival curves for mice with $\mathrm{HH}$-associated medulloblastoma. Tukey's multiple-comparison test (B and $\mathbf{C}$ ) and a log-rank test $(\mathbf{E})$ were used for statistical comparisons. abemaciclib blocked clonogenic growth of UW228 and ONS76 cells in a dose-dependent manner (Supplemental Figure 5, I and L), our findings that these cells lacked mechanisms underlying ciliary HH signal transduction and the UPR underscore the suboptimal nature of UW228 and ONS76 cells for studying oncogenic ciliary HH signaling. In support of this hypothesis, DAOY dCas9-KRAB suppression of RPL27A, which was implicated in resistance to CDK4/6 inhibitors in vitro (Supplemental Table 1), did not impair cell viability and validated that loss of other ribosome genes can mediate resistance to cell-cycle inhibition in $\mathrm{HH}$-associated medulloblastoma cells (Supplemental Figure 5, M and N). In sum, these data corroborate the essential, context-dependent cellular functions of ribosome genes (32) and reveal that ribosome suppression can confer a growth advantage in the context of cell-cycle inhibition in $\mathrm{HH}$-associated medulloblastoma.

To identify enzymes producing SMO-activating lipids that are influenced by ribosome gene expression and the UPR, we analyzed DAOY ${ }^{\mathrm{dCas}}$-KRAB cells stably expressing sgRNAs targeting RPL1O or sgNTCs by lipidomic mass spectrometry. Suppression of RPL1O increased cellular levels of cholesterol and 7-ketocholesterol (7k-C) (Figure 1M), which are produced by DHCR7 and HSD11ß2, respectively (33). DHCR7 promotes HH pathway activity by producing cholesterol (29), and we have shown that HSD11 22 drives HH-associated medulloblastoma by producing SMO-activating oxysterols (30). Suppression of RPL1O also increased 7k,27-hydroxycholesterol (7k,27-OHC) and 7,27-dihydroxycholesterol (7,27-DHC) (Figure 1M), both of which are byproducts of $7 \mathrm{k}-\mathrm{C}$, produced by HSD11 $\beta 2$, that bind to SMO and activate the HH pathway (30). Stable transduction of DAOY'as9 and DAOY ${ }^{\mathrm{dCas} 9-\mathrm{KRAB}}$ cells with sgRNAs targeting HSD11B2 demonstrated that genetic inactivation or suppression of HSD11B2 inhibited the $\mathrm{HH}$ transcriptional program and attenuated clonogenic growth of $\mathrm{HH}$-associated medulloblastoma cells compared with cells transduced with sgNTCs (Supplemental Figure 6). However, immunoblots revealed that suppression of RPL1O in DAOY ${ }^{\mathrm{dCas}-\mathrm{KRAB}}$ cells did not increase the expression of DHCR7 or HSD11ß2 proteins (Supplemental Figure 7A), and RNA-Seq of genetically engineered mouse models showed that enzyme transcripts were not increased in $\mathrm{HH}$-associated medulloblastomas lacking C $d k 6$ (Supplemental Figure 7B). These data suggest that the UPR regulates the activity, but not expression, of enzymes producing SMO-activating lipids, which has been demonstrated for other lipid synthases that are stimulated by UPR-mediated changes in ER membrane composition (34). Indeed, ER stress is known to activate lipogenesis through the UPR, which provides lipids for ER expansion that serve as precursors for lipid synthases localizing to the ER (35). In support of this hypothesis, 7-dehydroxycholesterol, which DHCR7 converts into cholesterol, was also increased in DAOY ${ }^{\mathrm{dCas}-\mathrm{KRAB}}$ cells stably expressing sgRNAs suppressing RPL1O compared with sgNTCs (Supplemental Figure 7C). 
Our discovery that SMO-activating lipid levels can increase in response to $\mathrm{CDK} 4 / 6$ inhibition suggests that combination molecular therapy against CDK6 and HSD11 $\beta 2$ may be an effective treatment for $\mathrm{HH}$-associated medulloblastomas. To test this hypothesis, we treated Math1-Cre SmoM2 ${ }^{C}$ mice with (a) vehicle control, (b) abemaciclib, (c) the HSD1132 inhibitor carbenoxolone (CNX) that penetrates the blood-brain barrier $(30,36)$, or (d) both abemaciclib and CNX. Combination molecular therapy reduced the histologic prevalence of malignant small, round blue cells that are characteristic of medulloblastoma and partially restored the internal architecture of cerebella when compared with vehicle control or molecular monotherapy (Figure 2A). Moreover, Math1-Cre SmoM2 ${ }^{C}$ medulloblastomas treated with combination molecular therapy showed a significant reduction in tumor weight compared with vehicle control or monotherapy (Figure 2B). CNX in combination with palbociclib, another small-molecule CDK4/6 inhibitor, was also more effective than monotherapy at blocking the growth medulloblastomas in the Math1-Cre Ptch1 ${ }^{\mathrm{C} / C}$ conditional mouse genetic model of $\mathrm{HH}$-associated medulloblastoma (Figure 2, C and D). To validate the specificity of our results, Math1-Cre, $S m o M 2^{c}, C d k 6^{K O}$, and $H s d 11 b 2^{f l}$ alleles were combined, and mice were monitored for survival from medulloblastomas expressing Cdk6, Hsd11b2, both, or neither (Figure 2E and Supplemental Figure 7). We previously reported that genetic inhibition of $\mathrm{Hsd} d 1 \mathrm{b2}$ decreases SMO-activating lipid levels, $\mathrm{HH}$ signaling, and growth of $\mathrm{HH}$-associated medulloblastoma in mice (30). Consistently, combined heterozygous genetic deletion of Cdk6 and Hsd11b2 modestly increased the length of survival from Math1-Cre SmoM2 ${ }^{C}$ medulloblastomas (median survival, $64 \pm 16$ days vs. $56 \pm 3$ days, $P$ = 0.006), and homozygous genetic deletion of Cdk6 and Hsd11b2 doubled the length of survival (median survival, $113 \pm 22$ days, $P=$ $7 \times 10^{-9}$ ), resulting in durable cures for $27 \%$ of mice versus $0 \%$ of mice with intact or heterozygous loss of Cdk6 or Hsd11b2 (Figure 2E and Supplemental Figure 8).

In conclusion, SMO-activating lipids underlie resistance to CDK4/6 inhibition in $\mathrm{HH}$-associated medulloblastoma, establishing a preclinical rationale for the use of combination molecular therapy to treat the most common malignant brain tumor in children. Our results reveal that pharmacologic inhibition of the cell cycle can be overcome by suppression of ribosomes or ribosome biogenesis genes, which activates the UPR to stimulate enzymes producing sterol and oxysterol lipids that activate SMO and sustain oncogenic $\mathrm{HH}$ signaling. As a proof of principle, we demonstrate that genetic or pharmacologic inhibition of HSD11 32 can enhance the efficacy of cell-cycle inhibitors for $\mathrm{HH}$-associated medulloblastoma, but it is possible that inhibition of other enzymes producing SMO-activating lipids may also provide a benefit to patients with $\mathrm{HH}$-associated medulloblastomas. Suppression of ribosome biogenesis has not been identified as a mechanism of resistance to cell-cycle inhibition in other cancers (37-39). Given the broad metabolic functions of ribosomes (20), including promotion of breast cancer metastasis (40), lipid homeostasis may influence resistance to pharmacologic inhibitors of the cell cycle in breast or other cancers.

\section{Methods}

Refer to the Supplemental Methods for details.

Data availability. All raw sequencing data can be accessed in the NCBI's Gene Expression Omnibus (GEO) database (GEO GSE164311).

Study approval. All animal protocols and experimental protocols were approved by the IACUC of UCSF (AN174769-02).

\section{Author contributions}

VD and DRR designed the study and analysis. Experiments were performed by VD, JH, AB, AC, ALK, PK, PT, AL and DRR. Data analysis was performed by $\mathrm{VD}, \mathrm{JH}, \mathrm{AB}, \mathrm{AC}, \mathrm{PT}, \mathrm{AL}$, and $\mathrm{DRR}$. The study was supervised by LX, JFR, and DRR. The manuscript was prepared by VD and DRR with input from all authors.

\section{Acknowledgments}

The authors thank S. John Liu, Harish N. Vasudevan, Rajat Rohatgi, Luke Gilbert, Davide Ruggero, Till Milde, and Jonathan Weissman for providing reagents and expertise. This study was supported by a St. Baldrick's Sumer Fellowship (to VD); NIH grant R01 HD092659 (to LX); NIH grant T32 GM007618 (to VD and AC); and the UCSF Physician Scientist Scholar Program and NIH grant K08 CA212279 (to DRR). Graphical abstract illustration credit: Noel Sirivansanti.

Address correspondence to: David R. Raleigh, UCSF Helen Diller Cancer Research Building, $14503^{\text {rd }}$ Street, HD481, San Francisco, California 94158, USA. Phone: 415.353.7175; Email: david. raleigh@ucsf.edu.
1. Ingham PW, et al. Mechanisms and functions of Hedgehog signalling across the metazoa. Nat Rev Genet. 2011;12(6):393-406.

2. Raleigh DR, Reiter JF. Misactivation of Hedgehog signaling causes inherited and sporadic cancers. J Clin Invest. 2019;129(2):465-475.

3. Garcia G, Raleigh DR, Reiter JF. How the ciliary membrane is organized inside-out to communicate outside-in. Curr Biol. 2018;28(8):R421-R434.

4. Sekulic A, Hoff Von D. Hedgehog pathway inhibition. Cell. 2016;164(5):831.

5. Yauch RL, et al. Smoothened mutation confers resistance to a Hedgehog pathway inhibitor in medulloblastoma. Science. 2009;326(5952):572-574.

6. Rudin CM, et al. Treatment of medulloblastoma with hedgehog pathway inhibitor GDC-0449.
N Engl J Med. 2009;361(12):1173-1178.

7. Robinson GW, et al. Irreversible growth plate fusions in children with medulloblastoma treated with a targeted hedgehog pathway inhibitor. Oncotarget. 2017;8(41):69295-69302.

8. Kieran MW, et al. Brain tumors: from childhood through adolescence into adulthood. J Clin Oncol. 2010;28(32):4783-4789.

9. Bhakta N, et al. The cumulative burden of surviving childhood cancer: an initial report from the St Jude Lifetime Cohort Study (SJLIFE). Lancet. 2017;390(10112):2569-2582.

10. O'Leary B, et al. Treating cancer with selective CDK4/6 inhibitors. Nat Rev Clin Oncol. 2016;13(7):417-430.

11. Raleigh DR, et al. Hedgehog signaling drives medulloblastoma growth via CDK6. JClin Invest.
2017;128(1):120-124.

12. Ivanov DP, et al. In vitro models of medulloblastoma: Choosing the right tool for the job. J Biotechnol. 2016;236:10-25.

13. Bialistoky T, et al. Functional analysis of NiemannPick disease type $\mathrm{C}$ family protein, NPC1a, in Drosophila melanogaster. Development. 2019;146(10):dev168427.

14. He M, et al. The kinesin-4 protein Kif7 regulates mammalian Hedgehog signalling by organizing the cilium tip compartment. Nat Cell Biol. 2014;16(7):663-672.

15. Rosengren T, et al. TSC1 and TSC2 regulate cilia length and canonical Hedgehog signaling via different mechanisms. Cell Mol Life Sci. 2018;75(14):2663-2680.

16. Li Z, et al. Loss of the FAT1 tumor suppressor 
promotes resistance to CDK4/6 inhibitors via the hippo pathway. Cancer Cell. 2018;34(6):893-905.

17. Cornell L, et al. MicroRNA-mediated suppression of the TGF- $\beta$ pathway confers transmissible and reversible CDK4/6 inhibitor resistance. Cell Rep. 2019;26(10):2667-2680.

18. Han YG, et al. Dual and opposing roles of primary cilia in medulloblastoma development. Nat Med. 2009;15(9):1062-1065.

19. Schüller U, et al. Acquisition of granule neuron precursor identity is a critical determinant of progenitor cell competence to form Shh-induced medulloblastoma. Cancer Cell. 2008;14(2):123-134.

20. Ruggero D, Pandolfi PP. Does the ribosome translate cancer? Nat Rev Cancer. 2003;3(3):179-192.

21. Adamson B, et al. A multiplexed single-cell CRISPR Screening platform enables systematic dissection of the unfolded protein response. Cell. 2016;167(7):1867-1882.

22. De Keersmaecker K, et al. Exome sequencing identifies mutation in CNOT3 and ribosomal genes RPL5 and RPL10 in T-cell acute lymphoblastic leukemia. Nat Genet. 2013;45(2):186-190.

23. Hofman IJF, et al. Low frequency mutations in ribosomal proteins RPL10 and RPL5 in multiple myeloma. Haematologica. 2017;102(8):e317-e320.

24. Sulima SO, et al. Ribosomal lesions promote oncogenic mutagenesis. Cancer Res.
2019;79(2):320-327.

25. Liu Y, et al. Ribosomal protein-Mdm2-p53 pathway coordinates nutrient stress with lipid metabolism by regulating MCD and promoting fatty acid oxidation. Proc Natl Acad Sci U S A. 2014;111(23):E2414-2422.

26. Pantazi A, et al. Inhibition of the 605 ribosome biogenesis GTPase LSG1 causes endoplasmic reticular disruption and cellular senescence. Aging Cell. 2019;18(4):e12981.

27. Lee A-H, et al. Regulation of hepatic lipogenesis by the transcription factor XBP1. Science. 2008;320(5882):1492-1496.

28. Sriburi R, et al. XBP1: a link between the unfolded protein response, lipid biosynthesis, and biogenesis of the endoplasmic reticulum. J Cell Biol. 2004;167(1):35-41.

29. Kinnebrew M, et al. Cholesterol accessibility at the ciliary membrane controls hedgehog signaling. Elife. 2019;8:e50051.

30. Raleigh DR, et al. Cilia-associated oxysterols activate smoothened. Mol Cell. 2018;72(2):316-327.

31. Kennedy D, et al. Methods for studying ER stress and UPR markers in human cells. Methods $\mathrm{Mol}$ Biol. 2015;1292(8):3-18.

32. Frakes AE, Dillin A. The UPR ${ }^{\text {ER: }}$ sensor and coordinator of organismal homeostasis. Mol Cell. 2017;66(6):761-771.
33. Russell DW. The enzymes, regulation, and genetics of bile acid synthesis. Annu Rev Biochem. 2003;72(1):137-174.

34. Sriburi R, et al. Coordinate regulation of phospholipid biosynthesis and secretory pathway gene expression in XBP-1(S)-induced endoplasmic reticulum biogenesis. J Biol Chem. 2007;282(10):7024-7034.

35. Bravo R, et al. Endoplasmic reticulum and the unfolded protein response: dynamics and metabolic integration. Int Rev Cell Mol Biol. 2013;301:215-290.

36. Paterson JM, et al. Genetic manipulation of 11beta-hydroxysteroid dehydrogenases in mice. Am J Physiol Regul Integr Comp Physiol. 2005;289(3):R642-R652.

37. Portman N, et al. Overcoming CDK4/6 inhibitor resistance in ER-positive breast cancer. Endocr Relat Cancer. 2019;26(1):R15-R30.

38. Pandey K, et al. Molecular mechanisms of resistance to CDK4/6 inhibitors in breast cancer: a review. Int J Cancer. 2019;145(5):1179-1188.

39. Formisano L, et al. Aberrant FGFR signaling mediates resistance to CDK4/6 inhibitors in $\mathrm{ER}^{+}$ breast cancer. Nat Commun. 2019;10(1):1373.

40. Ebright RY, et al. Deregulation of ribosomal protein expression and translation promotes breast cancer metastasis. Science. 2020;367(6485):1468-1473. 